\title{
Älter werden in Balance - Präventionspotenziale in Pflegeeinrichtungen
}

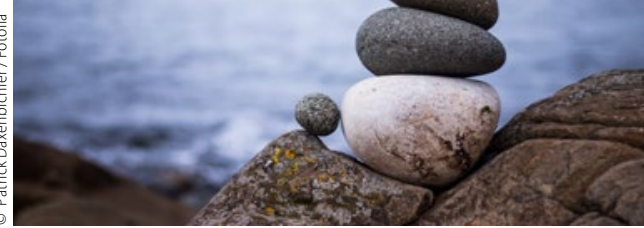

\begin{abstract}
Mit einem Gongschlag "für mehr Aufmerksamkeit", wie er sagte, eröffnete der Moderator und Arzt Dr. Eckart von Hirschhausen die 5. Bundeskonferenz "Körperlich und geistig aktiv bis ins hohe Alter", durchgeführt von der Bundeszentrale für gesundheitliche Aufklärung (BZgA), vom Bundesministerium für Gesundheit und vom Verband der Privaten Krankenversicherung (PKV).
\end{abstract}

Passenderweise am „Tag der älteren Generation“ am 5. April stellte die BZgA das „Lübecker Modell Bewegungswelten“ vor. Dieses soll als alltagsnahes und effektives Training zur Bewerbungsförderung in Pflegeheimen beitragen.

„Wer sich auch im Alter ausreichend bewegt, tut etwas dafür, länger selbstständig und geistig fit zu bleiben“, sagte Annette Widmann-Mauz, parlamentarische Staatssekretärin im Bundesgesundheitsministerium. So helfe ein körperlich aktiver Stil dabei, Erkrankungen vorzubeugen oder zu lindern. Bewegung solle dabei vor allem Freude machen und in den Alltag integriert und weitergeführt werden. „Immer mehr Menschen erreichen ein hohes Lebensalter, dies ist aber nicht gleichzusetzen mit Pflegebedürftigkeit", denn die meisten Älteren seien nicht angewiesen auf Pflegeangebote. Daher müsse man die Potenziale nutzen, um das Risiko, im Alter pflegebedürftig zu werden, zu minimieren.

\section{Nie zu früh und nie zu spät}

Dr. Heidrun Thaiss, Leiterin der BZgA, betonte: „Vieles, was uns schon in der frühen Kindheit beschäftigt, beschäftigt uns auch im Alter. Es lohnt sich daher, eine Brücke zu schlagen." Es sei nie zu früh und nie zu spät für Prävention und gesundheitsfördernde Maßnahmen, denn auch bei Menschen, die erst in höherem Alter beginnen, sich ausdauernd zu bewegen, ließe sich ein Wachstum der Telomere feststellen, also eine Verjüngung der Zellen.

Viele chronische Erkrankungen im Alter, etwa kardiovaskuläre Krankheiten, Schlaganfall oder Diabetes Typ 2 bis hin zur Erblindung seien nicht in jedem Fall unausweichlich. Dennoch ließen sich bei Menschen mit einer Vorstufe des Typ-2-Diabetes mit einem speziell auf die gestörte Glukoseintoleranz zugeschnittenen Sportprogramm in 60 Prozent der Fälle ein Ausbruch der Krankheit verhindern.

PKV-Direktor Volker Leienbach sagte dazu: „Unsere Aufgabe ist es, Pflegebedürftigkeit gar nicht erst entstehen zu lassen." Mit dem „Lübecker Modell Bewegungswelten“ könnten nun Pflegeheimbewohner innerhalb kurzer Zeit Kraft, Selbstständigkeit und Mobilität zurückerlangen. Darin werden bestimmte Übungen in Situationen aus dem Alltag eingebettet, wodurch sich nicht nur die Motivation zum Mitmachen erhöhen, sondern auch die Freude an der Bewegung steigen soll. In der Bewegungswelt „Ein Tag im Garten“ etwa ahmen die Teilnehmer körperliche Aktivitäten wie „Äpfel pflücken“ und „den Boden umgraben“ nach. Es entstehen ganze „Bewegungsgeschichten“. Erste Ergebnisse belegen laut BZgA, dass Mobilität, Koordination, Kognition und Lebensqualität der Teilnehmenden positiv beeinflusst werden konnten.

Die Pilotphase des Lübecker Modells hat im Februar 2016 und im April der Rollout in Berlin begonnen.

\section{„Bislang wollte keiner Verantwortung und Risiken übernehmen“}

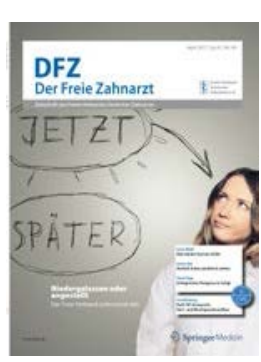

„Unter der Überschrift, Vorsicht! Angestellte Zahnärzte!' bezeichnet Bertram Steiner die ganz sicher in Kollegenkreisen geführte Diskussion über die Bereitschaft von jungen Zahnärztinnen und Zahnärzten, sich niederzulassen oder als Angestellte zu arbeiten und die daraus sich ergebende Meinung vieler Kolleginnen und Kollegen, dass unsere, Nachkommenschaft' nur sehr geringe Bereitschaft zur Selbstständigkeit mit allen ihren Nachteilen und Risiken zeigt, als,Quatsch!

Nach 44-jähriger Tätigkeit in eigener Praxis, die vergangenen neun Jahre zusammen mit meinem Sohn, muss ich leider feststellen, dass Herr Kollege Bertram Steiner an der Wirklichkeit vorbei schreibt. Mein Sohn und ich suchen schon seit vielen Monaten eine Nachfolge für mich, weil ich in den wohlverdienten $R u$ hestand gehen möchte. Bisher sind alle Versuche, eine geeignete Kollegin oder einen geeigneten Kollegen zu finden, als gescheitert zu bezeichnen. Warum? Weil bisher keiner der wenigen Bewerberinnen und Bewerber bereit war, später einmal Verantwortung und Risiken eines freien Berufes zu übernehmen. In der Regel wurde auf ein, Angestelltenverhältnis' gepocht, mit festen Arbeitszeiten (zum Beispiel 9 Uhr bis 12 Uhr und 15 Uhr bis $18 \mathrm{Uhr}$, Mittwochnachmittag und häufig auch Freitagnachmittag frei). Ein angebotener späterer Einstieg als Partnerin oder Partner wird durchweg abgelehnt. Bei den Gehaltsforderungen gelten aber keine Grenzen. Außerdem zeigt bereits die sehr geringe Anzahl von Bewerberinnen und Bewerbern, egal über welche von uns eingeschaltete Institution (Presse, LZK, KZV, Agentur für Arbeit usw.), das , so große Interesse der jungen Kolleginnen und Kollegen an einer Anstellung in einer Praxis. Die Wirtschaft und das Ausland sind mit ihren attraktiven Angeboten viel lukrativer.

Das sind meine und unsere Erfahrungen mit, Bewerbern' als Angestellte. Wer das als,Quatsch'bezeichnet, sollte sich einmal darum bemühen, einen angestellten Zahnarzt oder Sozius für seine Praxis zu bekommen!"

Dr. Eberhard Rusch, Felsberg-Gensungen 